\title{
Cognitive function in early onset schizophrenia: a selective review
}

\author{
Sophia Frangou* \\ Section of Neurobiology of Psychosis, Institute of Psychiatry, King's College London, London, UK
}

\section{Edited by:}

Vince D. Calhoun,

University of New Mexico, USA

Reviewed by:

Godfrey Pearlson, Yale University

School of Medicine, USA; Sanjir Kumra,

University of Minnesota, USA

\section{${ }^{*}$ Correspondence:}

Sophia Frangou, Section of

Neurobiology of Psychosis (P066),

Institute of Psychiatry,

King's College London, De Crespigny

Park, London SE5 8AF, UK.

e-mail: sophia.frangou@kcl.ac.uk
Schizophrenia is widely regarded as the clinical outcome of aberrant neurodevelopment caused by a combination of genetic and non-genetic factors. Early Onset Schizophrenia (EOS) manifests in childhood or adolescence and represents a more severe variant of the Adult Onset form of the disorder (AOS). EOS offers a unique opportunity of exploring the impact of disease related mechanisms on the developmental trajectory of cognitive function. The present review focused on the domains of general intellectual ability $(\mathrm{IQ})$, attention, executive function and memory. Significant methodological variability was noted across the different studies that examined these aspects of cognition in EOS patients. Despite this, a consistent pattern emergent from the data suggesting that (a) EOS patients compared to healthy children and adolescents show impairments of medium to large effect size in $\mathrm{IQ}$, attention, memory and executive function (b) despite increased clinical severity, the cognitive profile of EOS patients is comparable to that of AOS patients (c) healthy adolescents show age-related improvement in their ability to perform tests of attention, memory and executive function; this is not present in EOS patients thus resulting in increased age-related deviance in patients' performance. This apparent decline is mostly attributable to patients' failure to acquire new information and to use more sophisticated cognitive strategies.

Keywords: cognition, childhood, adolescent, schizophrenia, intelligence, memory, executive function, attention

\section{INTRODUCTION}

Early Onset Schizophrenia (EOS), regardless of whether it presents in childhood (childhood onset schizophrenia; COS) or adolescence (adolescent onset schizophrenia; AdOS) lies on the same diagnostic continuum with the adult onset (AOS) form of the disorder (Asarnow et al., 1994; Nicolson and Rapoport, 1999); longitudinal studies have confirmed the phenotypical continuity of EOS with adult onset cases since schizophrenia diagnosed in childhood and adolescent shows high diagnostic stability even over 40 years of follow-up (Remschmidt et al., 2007). Similarly brain imaging studies have also established a neurobiological continuum between early and adult onset schizophrenia (Thompson et al., 2001; Greenstein et al., 2006; Burke et al., 2008; Kyriakopoulos et al., 2009).

However, EOS is associated with more severe clinical course (Werry et al., 1991; Eggers and Bunk, 1997), greater premorbid abnormalities (Watkins et al., 1988; Cannon et al., 2002; Vourdas et al., 2003), greater genetic loading (Asarnow, 1999) and developmental deviance (Hollis, 1995; Vourdas et al., 2003).

For more than two decades, the neurodevelopmental model has been the prevailing explanatory theory for the aetiology of schizophrenia. In its simplest form this model posits that schizophrenia is the behavioural outcome of aberrant neurodevelopment that begins long before the onset of clinical symptoms and is caused by a combination of environmental and genetic factors (Rapoport et al., 2005). In this context the study of EOS offers an opportunity to explore how disease related mechanisms may impact on facets of cognitive function and their developmental trajectories. This article presents a selective review of the relevant literature focusing on four key aspects of cognition, namely general intellectual ability, attention, executive function and memory.

\section{GENERAL INTELLECTUAL ABILITY}

In clinical EOS samples general intellectual ability has been most commonly assessed using the Intelligence Quotient (IQ) obtained from the full or short versions of the Wechsler Intelligence Scale for Children-Revised (WISC-R) (Wechsler, 1974) (ages 6-16 years) or Wechsler Adult Intelligence Scale - Revised (WAIS-R) (Wechsler, 1981) (17 years and older). In contrast epidemiological studies have used a variety of measures, their findings however appear consistent across studies and independent of the choice of test (Woodberry et al., 2008).

\section{CROSS-SECTIONAL STUDIES}

General Intellectual ability is compromised in EOS. Mean IQ in EOS patients has been consistently found to be about 1-1.5 standard deviations below the normative mean (Bedwell et al., 1999; Kumra et al., 2000; Kravariti et al., 2003b; Gochman et al., 2005; Fagerlund et al., 2006; White et al., 2006).

\section{LONGITUDINAL STUDIES}

\section{Premorbid and prodromal phase}

Epidemiological studies of healthy children and adolescents have established that (a) deviance from cognitive norm for general intellectual ability significantly increases the risk of schizophrenia and, that (b) schizophrenia patients as a group have lower premorbid IQ scores (Cannon et al., 2002; Reichenberg et al., 2005). This has been 
confirmed further by a meta-analysis by Woodberry et al. (2008); the effect size of premorbid IQ decrement was medium but independent of any methodological considerations relating to sample characteristics such as age at the time of testing. They also reported IQ decline associated with the onset of psychotic symptoms. Bedwell et al. (1999) were amongst the first to report this observation in COS patients from the National Institute of Mental Health (NIMH) cohort. They noted a decline in the age-corrected (but not raw) IQ scores which began about 2 years prior to the onset of frank psychosis and continued for up to 2 years post-onset. The authors suggested that this apparent decline was related not to loss of function but to the inability of EOS patients to acquire new skills and information in line with their healthy adolescent counterparts.

\section{Longitudinal, post-psychosis onset studies}

Most of the evidence available suggests that general intellectual ability in EOS remains largely stable after the onset of psychosis. Gochman et al. (2005) analysed IQ data from 70 COS patients from the NIMH cohort. With the exception of 13 patients for whom earlier IQ scores were available, baseline IQ measures were obtained after the onset of psychosis during the patients' initial assessment at the NIMH. Patients were followed up for 8 years with further IQ evaluations at 2-year intervals. Over this lengthy follow-up period, IQ scores fluctuated but there was no significant overall change. In contrast to earlier observations by Bedwell et al. (1999), in this larger NIMH sample raw scores for the information, comprehension and picture arrangement subscales increased significantly over time. Frangou et al. (2008) reached very similar conclusions when examining longitudinal IQ data from 20 EOS patients and their matched healthy controls from the Maudsley Early Onset Schizophrenia Study. In this cohort, IQ was assessed on two occasions, once within the first two years from the onset of psychosis and then on average 4 years later. Both patients and controls showed a small but statistically significant improvement in their IQ score between baseline and follow-up assessments.

\section{COMPARISON TO ADULT ONSET SCHIZOPHRENIA}

Despite increased illness EOS patients appear to have comparable deficits to AOS patients with respect of general intellectual ability. A study of Indian EOS patients is the only one to have found greater IQ impairment in COS than adult onset schizophrenia with AdOS patients occupying an intermediate position (Biswas et al., 2006). In all other studies no significant differences in IQ were reported between COS, AdOS (Rhinewine et al., 2005) and AOS samples (White et al., 2006). White et al. (2006) have undertaken the only study to date to directly contrast the cognitive profiles of EOS of AOS patients. Their study sample comprised of 49 adolescents with COS or AdOS, 139 AOS patients with first episode schizophrenia and 272 healthy volunteers (32 adolescents and 240 adults). General intellectual function was evaluated with the WAIS-R which yielded nearly identical IQ scores for both patient groups; these were approximately 1 standard deviation below the mean IQ of the healthy controls.

\section{ATTENTION}

The nature and degree of attentional impairment in EOS remain unclear due to significant between-study variability. This probably relates both to the sensitivity of the neuropsychological tests used and the size and clinical features of study samples. Although attention underpins cognitive function in general there are several tests that are conventionally construed as relatively specific assays of different aspects of attention. In the literature reviewed here attention was commonly assessed using the Trails Making Test (TMT; Reitan, 1995) and particular subtests of the WISC-R and WAIS-R. Versions of the Continuous Performance Test (CPT) and the dichotic listening test were used to evaluate sustained and selective attention respectively.

\section{CROSS-SECTIONAL STUDIES}

Kumra et al. (2000) reported that COS patients from the NIMH cohort showed marked attentional dysfunction. Their observation was based on data from the TMT and the Coding, Digit Symbol and Digit Span subtests of the WAIS-R or WISC-R which were used to form a composite measure of attention. Patients scored about two standard deviations below the normative mean. Øie and Rund (1999) reported that their sample of 19 EOS patients were not significantly impaired in sustained attention, as measured by the Degraded Symbol CPT (DS-CPT; Nuechterlein and Dawson, 1984), or in selective attention as assessed using a dichotic listening task. The TMT and the Digit Symbol subtest of the WISC-R were used to form a summary measure of "visuomotor processing and attention". Patients scored about two standard deviations below the control mean on this particular variable. Brickman et al. (2004) grouped the Digit Span subtest of the Wechsler intelligence scales and the TMT to form a composite variable for attention. Their sample comprised of 39 never medicated adolescents with first episode psychosis whose score was nearly 3 standard deviations below that of controls on this particular variable. Additionally, an impairment of nearly two standardar deviations compared to controls was noted in a version of the Digit Span in a sample of 34 young people with schizophrenia and related disorders (Karatekin et al., 2008). In contrast, data from the Maudsley Early Onset Schizophrenia Study did not reveal attentional deficits in EOS patients compared to matched controls (Kravariti et al., 2003a). Attention in this study was measured using the Attention/Concentration factor of the WMS-R and selective attention was evaluated using a dual task paradigm.

\section{LONGITUDINAL, POST-PSYCHOSIS ONSET STUDIES}

The issue of age-related changes in attention in EOS patients has been explored in three different samples. Thaden et al. (2006) administered the Continuous Performance Test-Identical Pairs (IPCPT; Cornblatt et al., 1988) to 59 EOS adolescents and 55 matched normal controls. Across the age span of the sample (10-20 years of age) healthy adolescents improved in task performance and this was particularly true for task conditions with high processing load. This was not present in patients, suggesting a failure in the developmental trajectory that underpins increased attentional capacity with age. Frangou et al. (2008) and Øie and colleagues (Øie and Hugdahl, 2008; Øie et al., 2008) examined longitudinal data from the Maudsley Early Onset Schizophrenia Study and from a Norwegian sample of EOS patients followed-up over an average period of 4 and 13 years respectively. Both studies reported that EOS patients showed a decline in attentional measures. Their agematched healthy counterparts showed age-related improvements in attentional processing which was absent in EOS patients. 


\section{COMPARISON TO ADULT ONSET SCHIZOPHRENIA}

When contrasting EOS and AOS patients to healthy controls, White et al. (2006) combined four tasks to compose the summary variable corresponding to the domain of attention; these were the CPT (Conners and Staff, 1995), the Circle A Letter-cancellation task (Talland and Schwab, 1964), the Stroop Color and Word Test (Golden, 1978), and the TMT. Both EOS and AOS patients performed worse than healthy controls in this domain but there was no difference between the two patient groups.

\section{EXECUTIVE FUNCTION}

Executive function emerged as a concept in neuroscience mostly based on the association between frontal lobe integrity and behaviours such as problem solving, response inhibition, strategy development and implementation and working memory. Elliott (2003) proposed that executive functioning can be defined as the complex process of coordinating multiple sub-processes to achieve a particular goal. Given that multiple facets of cognition are currently considered constituent aspects of executive function it is not surprising that many different tests have been used to examine executive function in EOS. There are two issues that become apparent when reviewing the literature which are not specific to EOS but are pervasive in the field of cognitive research. Firstly, individual studies only test a limited range of cognitive processes that comprise executive function. However the presentation and interpretation of the results often refers to executive function as a whole thus potentially obscuring informative differences regarding aspects of executive function that may be affected or preserved. Secondly, there is no consensus with respect to tests of executive control of attention or memory which are attributed to specific cognitive domains depending on the authors' preferences.

\section{CROSS-SECTIONAL STUDIES}

Kenny et al. (1997) compared 17 EOS patients to an equal number of matched healthy adolescents using a broad neurocognitive battery. Test relevant to executive function included the tests of abstraction and perseveration, namely the Wisconsin Card Sorting Test (WCST; Heaton et al., 1993 and the maze subtest of the WISC$\mathrm{R}$, the Trigram Recall with Interference Test (Baddeley, 1986) and the coding subtest of the WISC-R (both considered tests of working memory) as well the Controlled Oral Word Retrieval (Benton, 1968) and Category Instance Retrieval Tests (Perret, 1974) that measure verbal fluency. The biggest effect size was noted for working memory (effect size 1.65-1.79), followed by abstraction and perseveration (effect size $0.61-0.68$ ) and category retrieval (effect size 0.66). Øie and Rund (1999) in their sample of adolescent schizophrenics also found deficits in the WCST. Similar deficits in abstraction and cognitive set were also reported by the NIMH group (Kumra et al., 2000). In the Maudsley Early Onset schizophrenia study executive function was assessed using the Tower of London Test (TOL; Shallice, 1982) to measure planning and problem-solving and the Executive Golf task to measure spatial working memory (Kravariti et al., 2003a). In the TOL patients showed deficits in planning accuracy and reduced subsequent planning time (a measure of time spent planning and thinking about the next problem solving move), which may suggest greater impulsivity. This pattern was also observed by Fagerlund et al.
(2006). Deficits in spatial working memory related mostly to EOS patients' inability to generate an efficient strategy and less so in maintaining information in working memory.

\section{LONGITUDINAL STUDIES}

Cervellione et al. (2007) found adolescent schizophrenic patients to have significant impairment in executive function which remained unchanged over a 2-year follow-up period. Similarly, Frangou et al. (2008) evaluated the stability of executive function in the Maudsley Early Onset Schizophrenia study with respect to planning as problem solving as reflected by the TOL. Although planning accuracy was lower in EOS patients, both groups' maintained the same level of performance over the 4-year follow-up period. Øie et al. (2008) focused on abstraction and perseveration using the WCST. The performance of EOS patients did not change over the 13-year followup period but they failed to show the improvements in perseverative errors that were noted in their healthy counterparts.

\section{COMPARISON TO ADULT ONSET SCHIZOPHRENIA}

White et al. (2006) examined two aspects of executive function, namely working memory and problem solving when comparing EOS to AOS patients and to controls. The composite measure of working memory comprised the arithmetic and digit span subtests from the WAIS-R and it was the only domain where EOS patients underperformed compared to AOS patients. The functional domain of problem solving included test performance on the Wisconsin Card Sorting Test (WCST) (Heaton et al., 1993), the abstractions subtest from the Shipley Institute of Living Scale (Shipley, 1946) and the comprehension, similarities, picture completion and picture arrangement subtests from the WAIS-R. No differences between the patients groups were noted for the above variables.

\section{MEMORY}

Memory impairment is consistently reported in schizophrenia regardless of age of onset. Most studies assessing memory and learning in EOS have used versions of the Wechsler Memory Scales while the California Verbal Learning test (CVLT) (Delis et al., 1994) or similar list learning tasks have been widely used to measure verbal learning. Visual memory and learning are less commonly assessed, with the Kimura Recurring Figures Test (Kimura, 1980) being the most popular instrument employed.

\section{CROSS-SECTIONAL STUDIES}

Kenny et al. (1997) were amongst the first to report significant impairment in verbal memory and leaning in AOS patients compared to healthy adolescents. In their study verbal memory and learning were assessed using list learning tests (Buschke and Fuld, 1974) while immediate and delayed recall were assessed using the Logical Memory I and II subtests of the WMS-R (Wechsler, 1987). EOS patients underperformed in all measures of verbal learning and memory and in delayed (effect size range 0.68-0.96) but not immediate recall. The study by $\varnothing$ ie and Rund (1999) suggested that visual memory (assessed using the Kimura Recurring Figures Test) is also significantly impaired in EOS; the performance of their sample of AOS patients was on average two standard deviations below that of healthy adolescents. Ueland et al. (2004) also observed significant visual memory impairment in adolescents with schizophrenia 
spectrum disorder also using the Kimura Recurring Figures Test. However, visual memory (as assessed with the Wechsler Memory Scale-Revised; WMS-R) was only minimally affected in EOS patients from the Maudsley Early Onset Schizophrenia Study (Kravariti et al., 2003b) while significant impairment was again noted for verbal memory. Brickman et al. (2004) confirmed this finding in a sample of 29 never-medicated first-episode adolescents with psychosis where verbal memory and learning was assessed with a list learning task and was found to be significantly impaired in patients compared to controls. A similar finding was also reported by Landrø and Ueland (2008) who also examined verbal memory and learning using a list learning test when comparing 21 adolescents with schizophrenia spectrum disorders to healthy adolescents. Furthermore, the authors observed that the impairment primarily affected learning rather than recognition or recall. Roofeh et al. (2006) used the CVLT to further delineate the nature of the verbal memory and learning deficits in adolescents with schizophrenia and schizophrenia spectrum disorders. They found large impairments in patients compared to healthy controls in verbal learning, immediate and delayed recall, recognition memory and consistency of recall, but not in measures of rate of forgetting, proactive or retroactive interference or serial order effects in recall such as primacy and recency. Furthermore, in healthy individuals there was an age related switch in encoding and retrieval from serial recall to semantic clustering. Young patients with EOS and spectrum disorders failed to make this developmental change in memory organisation and retrieval.

\section{LONGITUDINAL, POST-PSYCHOSIS ONSET STUDIES}

Cervellione et al. (2007) found verbal learning and memory (as assessed with the CVLT) to be impaired in adolescents with schizophrenia but stable over a 2 -year follow-up period. However, in the 4-year follow-up of the EOS sample from the Maudsley Early Onset Schizophrenia Study (Frangou et al., 2008), patients showed evidence of decline in the verbal memory index of the WMS-R while healthy adolescents performed at the same level on both assessments. Careful examination revealed that this decline affected primarily acquisition of new information rather than consolidation and recall. Øie et al. (2008) reached the same conclusions using the CVLT; over a 13-year follow-up period healthy controls showed no change in their performance while EOS patients' performance in the total learning trials declined.

\section{COMPARISON TO ADULT ONSET SCHIZOPHRENIA}

According to Basso et al. (1997) verbal memory performance differentiated AdOS from AOS patients and controls being significantly more impaired in AdOS particularly in measures of immediate

\section{REFERENCES}

Asarnow, J. R., Tompson, M. C., and Goldstein, M. J. (1994). Childhoodonset schizophrenia: a follow-up study. Schizophr. Bull. 20, 599-617.

Asarnow, R. F. (1999). Neurocognitive impairments in schizophrenia: a piece of the epigenetic puzzle. Eur. Child Adolesc. Psychiatry 8(Suppl. 1), 5-8.

Baddeley, A. (1986). Working Memory. New York, Oxford University Press.
Basso, M. R., Nasrallah, H. A., Olson, S. C., and Bornstein, R. A. (1997). Cognitive deficits distinguish patients with adolescent- and adultonset schizophrenia. Neuropsychiatry Neuropsychol. Behav. Neurol. 10, 107-112.

Bedwell, J. S., Keller, B., Smith, A. K., Hamburger, S., Kumra, S., and Rapoport, J. L. (1999). Why does postpsychotic IQ decline in

recall. White et al. (2006) compared EOS and AOS patients to matched controls on two composite measures of verbal and nonverbal memory. The former included variables from a list learning test, the Rey Auditory Verbal Learning Test (RAVLT; Rey, 1958) and the logical memory subtest of the WMS-R. The latter included the immediate and delayed recall trials of the Rey-Osterrieth Complex Figure Test (ROCFT; Rey, 1941; Osterrieth, 1944) and the total number correct from the Benton Visual Retention Test-Revised (BVRT-R; Benton, 1974). The two patient groups did not differ in either verbal or non-verbal memory.

\section{CONCLUDING REMARKS}

This review examined the cognitive profile of EOS patients in terms of general intellectual ability, attention, executive function and memory. Despite significant differences in sample characteristics and cognitive tests employed a remarkably consistent pattern emerges from the overall literature. The evidence reviewed suggests that (a) EOS patients are impaired in all four domains examined (b) the degree of impairment in all four domains is comparable to that seen in AOS patients with the possible exception of working memory where EOS patients may be more impaired (c) there is little evidence for decline in any of the four domains, at least for up to 13 years post illness onset and (d) increased deviance in EOS patients as they moved into adulthood was noted for attention, memory and executive function but this was mostly due to patients' failure to show the age-related improvements in these domains compared to their healthy counterparts.

Speculation as to the potential mechanisms underlying the cognitive patterns identified is beyond the resolution of the data reviewed here. Additionally inferences about neural systems based solely on behavioural performance on cognitive tests can only be made with caution. However, it could be argued that, in EOS, the combination of relative stability in cognitive "dysfunction" coupled with lack of age-related improvement in cognitive abilities resonates with brain imaging findings relating to the brain's maturational trajectory. Longitudinal studies of normally developing individuals have shown that primary motor and sensory areas mature early while areas subserving more complex functions follow a back-to-front pattern starting at the parietal lobes and progressing towards frontal and temporal regions (Gogtay et al., 2004; Sowell et al., 2004). Recent longitudinal studies suggest in EOS patients grey matter maturation shows an abnormal trajectory with lower and later peaks than for healthy subjects (Douaud et al., 2009). This altered brain developmental time course is in line with the abnormal developmental course suggested by cognitive data.

childhood-onset schizophrenia? Am. J. Psychiatry 156, 1996-1997.

Benton, A. L. (1968). Differential behavioural effects in frontal lobe disease. Neuropsychologia 6, 53-60.

Benton, A. L. (1974). The Revised Visual Retention Test: Clinical and Experimental Applications, 4th edn. New York, Psychological Corporation. Biswas, P., Malhotra, S., Malhotra, A., and Gupta, N. (2006). Comparative study of neuropsychological correlates in schizophrenia with onset in childhood, adolescence and adulthood. Eur. Child Adolesc. Psychiatry 15, 360-366.

Brickman,A.M.,Buchsbaum,M.S.,Bloom, R., Bokhoven, P., Paul-Odouard, R., Haznedar, M. M., Dahlman, K. L., Hazlett, E. A., Aronowitz, J., Heath, D., and Shihabuddin, L. (2004). Neuropsychological functioning in first-break, never-medicated 
adolescents with psychosis. J. Nerv. Ment. Dis. 192, 615-622.

Burke, L., Androutsos, C., Jogia, J., Byrne, P., and Frangou, S. (2008). The Maudsley Early Onset Schizophrenia Study: the effect of age of onset and illness duration on fronto-parietal gray matter. Eur. Psychiatry 23, 233-236.

Buschke, H., and Fuld, P. A. (1974). Evaluating storage, retention and retrieval in disordered memory and learning. Neurology 11, 1019-1025.

Cannon, M., Caspi, A., Moffitt, T. E., Harrington, H., Taylor, A., Murray, R. M., and Poulton, R. (2002). Evidence for early-childhood, pandevelopmental impairment specific to schizophreniform disorder: results from a longitudinal birth cohort. Arch. Gen. Psychiatry 59, 449-456.

Cervellione, K. L., Burdick, K. E., Cottone, J. G., Rhinewine, J. P., and Kumra, S. (2007). Neurocognitive deficits in adolescents with schizophrenia: longitudinal stability and predictive utility for short-term functional outcome. J. Am. Acad. Child Adolesc. Psychiatry $46,867-878$.

Conners, C. K., and Staff, M. H. S. (1995). Conners' Continuous Performance Test. MHS, Toronto.

Cornblatt, B. A., Risch, N. J., Faris, G., Friedman, D., and ErlenmeyerKimling, L. (1988). The continuous performance test, identical pairs version (CPT-IP): new findings about sustained attention in normal families. Psychiatry Res. 26, 223-238.

Delis, D., Kramer, J., Kaplan, E., and Ober, B. (1994). California Verbal Learning Test-Children's Version (CVLT-C) Manual. San Antonio, Psychological Corporation.

Douaud, G., Mackay, C., Andersson, J., James, S., Quested, D., Ray, M. K., Connell, J., Roberts, N., Crow, T. J., Matthews, P. M., Smith, S., and James, A. (2009). Schizophrenia delays and alters maturation of the brain in adolescence. Brain 132(Pt. 9), 2437-2448.

Eggers, C., and Bunk, D. (1997). The long-term course of childhood-onset schizophrenia: a 42 -year followup. Schizophr. Bull. 23, 105-117.

Elliott, R. (2003). Executive functions and their disorders. Br. Med. Bull. 65, 49-59.

Fagerlund, B., Pagsberg, A. K., and Hemmingsen, R. P. (2006). Cognitive deficits and levels of IQ in adolescent onset schizophrenia and other psychotic disorders. Schizophr. Res. $8,30-39$.

Frangou, S., Hadjulis, M., and Vourdas, A. (2008). The Maudsley early onset schizophrenia study: cognitive function over a 4-year follow-up period. Schizophr. Bull. 34, 52-59.
Gochman, P. A., Greenstein, D., Sporn, A., Gogtay, N., Keller, B., Shaw, P., and Rapoport, J.L. (2005). IQ stabilization in childhood-onset schizophrenia. Schizophr. Res. 77, 271-277.

Gogtay, N., Giedd, J. N., Lusk, L., Hayashi, K. M., Greenstein, D., Vaituzis, A. C., Nugent T. F., III, Herman, D. H., Clasen, L. S., Toga, A. W., Rapoport, J. L., and Thompson, P. M. (2004). Dynamic mapping of human cortical development during childhood through early adulthood. Proc. Natl. Acad. Sci. U.S.A. 101, 8174-8179.

Golden, C. J. (1978). Stroop Color and Word Test: A Manual for Clinical and Experimental Users. Chicago, Stoelting Company.

Greenstein, D., Lerch, J., Shaw, P., Clasen, L., Giedd, J., Gochman, P., Rapoport, J., and Gogtay, N. (2006). Childhood onset schizophrenia: cortical brain abnormalities as young adults. J. Child. Psychol. Psychiatry 47, 1003-1012.

Heaton, R. K., Chelune, G., Talley, J.L., Kay, G., and Curtiss, G. (1993). Wisconsin Card Sorting Test Manual Revised and Expanded. Odessa, FL, Psychological Assessment Resources.

Hollis, C. (1995). Child and adolescent (juvenile onset) schizophrenia. A case control study of premorbid developmental impairments. Br. J. Psychiatry 166, 489-495.

Karatekin, C., White, T., and Bingham, C. (2008). Divided attention in youthonset psychosis and attention deficit/ hyperactivity disorder. J. Abnorm. Psychol. 117, 881-895.

Kenny, J. T., Friedman, L., Findling, R. L., Swales, T. P., Strauss, M. E., Jesberger, J. A., and Schulz, S. C. (1997). Cognitive impairment in adolescents with schizophrenia. Am. J. Psychiatry 154, 1613-1615.

Kimura, D. (1980). Kimura's RecurringFigures-Test: a normative study.J. Clin. Psychol. 2, 465-467.

Kravariti,E., Morris, R. G., Rabe-Hesketh, S., Murray, R. M., and Frangou, S. (2003a). The Maudsley early-onset schizophrenia study: cognitive function in adolescent-onset schizophrenia. Schizophr. Res. 65, 95-103.

Kravariti, E., Morris, R. G., Rabe-Hesketh, S., Murray, R. M., and Frangou, S. (2003b). The Maudsley early onset schizophrenia study: cognitive function in adolescents with recent onset schizophrenia. Schizophr. Res. 61, 137-148.

Kumra, S., Wiggs, E., Bedwell, J., Smith, A. K., Arling, E., Albus, K., Hamburger, S. D., McKenna, K., Jacobsen, L. K., Rapoport, J. L., and Asarnow, R. F. (2000). Neuropsychological deficits in pediatric patients with childhoodonset schizophrenia and psychotic disorder not otherwise specified. Schizophr. Res. 42,135-144.
Kyriakopoulos, M., Perez-Iglesias, R., Woolley, J. B., Kanaan, R. A., Vyas, N. S., Barker, G. J., Frangou, S., and McGuire, P. K. (2009). Effect of age at onset of schizophrenia on white matter abnormalities. Br. J. Psychiatry 195 346-353.

Landrø, N. I., and Ueland, T. (2008). Verbal memory and verbal fluency in adolescents with schizophrenia spectrum disorders. Psychiatry Clin. Neurosci. 62, 653-661.

Nicolson, R., and Rapoport, J. L. (1999). Childhood-onset schizophrenia: rare but worth studying. Biol. Psychiatry 46 , 1418-1428.

Nuechterlein, K. H., and Dawson, M. E. (1984). Information processing and attentional functioning in the developmental course of schizophrenic disorders. Schizophr. Bull. 10, 160-203.

Øie, M., and Hugdahl, K. (2008). A 10-13 year follow-up of changes in perception and executive attention in patients with early-onset schizophrenia: a dichotic listening study Schizophr. Res. 106, 29-32.

Øie, M., and Rund, B. R. (1999). Neuropsychological deficits in adolescent-onset schizophrenia compared with attention deficit hyperactivity disorder. Am. J. Psychiatry 156, 216-222.

Øie, M.,Sundet, K., and Rund, B. R. (2008). Neurocognitive decline in early-onset schizophrenia compared with ADHD and normal controls: evidence from a 13-year follow-up study. Schizophr. Bull. doi: 10.1093/schbul/sbn127.

Osterrieth, P. A. (1944). Le test de copie d'une figure complex: Contribution a l'etude de la perception et de la memoire. Arch. Psychol. 30, 286-356.

Perret, E. (1974). The left frontal lobe of man and the suppression of habitual responses in verbal categorical behaviour. Neuropsychologia 12 323-330.

Rapoport, J. L., Addington, A. M., and Frangou, S. (2005). The neurodevelopmental model of schizophrenia: update 2005. Mol. Psychiatry 10, 434-449.

Reichenberg, A., Weiser, M., Rapp, M. A., Rabinowitz, J., Caspi, A., Schmeidler J., Knobler, H. Y., Lubin, G., Nahon, D., Harvey, P. D., and Davidson, M (2005). Elaboration on premorbid intellectual performance in schizophrenia: premorbid intellectual decline and risk for schizophrenia. Arch. Gen. Psychiatry 62, 1297-1304.

Reitan, R. M. (1995). The relation of the trail making test to organic brain damage. J. Consult. Psychol. 19, 393-394.

Remschmidt,H., Martin, M., Fleischhaker C., Theisen, F. M., Hennighausen, K., Gutenbrunner, C., and Schulz, E. (2007). Forty-two-years later: the outcome of childhood-onset schizophrenia. J. Neural Transm. 114, 505-512.
Rey, A. (1941). L'examen psychologique dans les cas d'encephalopathie traumatique. Arch. Psychol. 28, 286-340.

Rey, A. (1958). L'examen clinique en psychologie. Paris, Presse Universitaire de France.

Rhinewine, J. P., Lencz, T., Thaden, E. P., Cervellione, K. L., Burdick, K. E., Henderson, I., Bhaskar, S., Keehlisen, L., Kane, J., Kohn, N., Fisch, G. S., Bilder, R. M., and Kumra, S. (2005). Biol. Psychiatry 58, 705-712.

Roofeh, D., Cottone, J., Burdick, K. E., Lencz, T., Gyato, K., Cervellione, K. L., Napolitano, B., Kester, H., Anderson, B., and Kumra, S. (2006). Deficits in memory strategy use are related to verbal memory impairments in adolescents with schizophrenia-spectrum disorders. Schizophr. Res. 85, 201-212.

Shallice, T. (1982). Specific impairments of planning. Philos. Trans. R. Soc. Lond. 298, 199-209.

Shipley, W. C. (1946). Institute of Living Scale. Los Angeles, Western Psychological Services.

Sowell, E. R., Thompson, P. M., Leonard, C. M., Welcome, S. E., Kan, E., and Toga, A. W. (2004). Longitudinal mapping of cortical thickness and brain growth in normal children. $J$. Neurosci. 24, 8223-8231.

Talland, G. A., and Schwab, R. S. (1964). Performance with multiple sets in Parkinson's disease. Neuropsychologia 2, 45-53.

Thaden, E., Rhinewine, J. P., Lencz, T., Kester, H., Cervellione, K. L., Henderson, I., Roofeh, D., Burdick, K. E., Napolitano, B., Cornblatt, B. A., and Kumra, S. (2006). Early-onset schizophrenia is associated with impaired adolescent development of attentional capacity using the identical pairs continuous performance test. Schizophr. Res. 81, 157-166.

Thompson, P. M., Vidal, C., Giedd, J. N. Gochman, P., Blumenthal, J., Nicolson, R., Toga, A. W., and Rapoport, J. L. (2001). Mapping adolescent brain change reveals dynamic wave of accelerated gray matter loss in very earlyonset schizophrenia. Proc. Natl. Acad. Sci. U.S.A. 98, 11650-11655.

Ueland, T., Øie, M., Inge Landrø, N., and Rund, B.R. (2004).Cognitive functioning in adolescents with schizophrenia spectrum disorders. Psychiatry Res. 126, 229-239.

Vourdas, A., Pipe, R., Corrigall, R., and Frangou, S. (2003). Increased developmental deviance and premorbid dysfunction in early onset schizophrenia. Schizophr. Res. 62, 13-22.

Watkins, J.M.,Asarnow, R. F., and Tanguay, P. E. (1988). Symptom development in childhood onset schizophrenia. 
J. Child. Psychol. Psychiatry 29, Werry, J. S., McClellan, J. M., and Chard, 865-878.

Wechsler, D. (1974). Manual for the Wechsler intelligence scale for children - Revised. New York, Psychological Corporation.

Wechsler, D. (1981). Wechsler Adult Intelligence Scale - Revised Manual. Cleveland, $\mathrm{OH}$, The Psychological Corporation.

Wechsler, D. (1987). Wechsler Memory Scale - Revised. New York, Psychological Corporation, Harcourt Brace Jovanovich.
Woodberry, K. A., Giuliano, A. J., and Seidman, L. J. (2008). Premorbid IQ in schizophrenia: a meta-analytic review. Am. J. Psychiatry 165, 579-587.

Conflict of Interest Statement: The author declares that the research was conducted in the absence of any commercial or financial relationships that could be construed as a potential conflict of interest.

Received: 08 June 2009; paper pending published: 21 October 2009; accepted:
29 December 2009; published online: 29 January 2010.

Citation: Frangou S (2010) Cognitive function in early onset schizophrenia: a selective review. Front. Hum. Neurosci. 3:79. doi: 10.3389/neuro.09.079.2009

Copyright () 2010 Frangou. This is an open-access article subject to an exclusive license agreement between the authors and the Frontiers Research Foundation, which permits unrestricted use, distribution, and reproduction in any medium, provided the original authors and source are credited. 